Griffiths, M. \& Stanier, R. Y. (1956). J. gen. Microbiol. 14, 698-715

\title{
Some Mutational Ghanges in the Photosynthetic Pigment System of Rhodopseudomonas spheroides
}

\author{
BY MARY GRIFFITHS AND R. Y. STANIER \\ Department of Bacteriology, University of California, Berkeley, \\ California, U.S.A.
}

SUMMARY : Some 50 mutants, which differ from the wild type in the nature of their pigment systems, were isolated. from the non-sulphur purple bacterium, Rhodopseudomonas spheroides. They fall into five main groups. The group of colourless mutants is incapable of photosynthetic growth, and devoid both of chlorophyll and carotenoids. The remaining groups still contain bacteriochlorophyll, but differ chemically from the wild type and from one another in their carotenoid pigments; all are capable of photosynthetic growth. The wild type contains two principal carotenoids, one red and one yellow. The dark red mutants contain both wild-type carotenoids and traces of a new red carotenoid. The brown mutants contain approximately equal amounts of the wild-type yellow carotenoid and of neurosporene, together with traces of the wild-type red carotenoid. The green mutants contain neurosporene and a dihydroxy derivative, but neither of the wild-type pigments. The blue-green mutants contain phytoene, a colourless polyene, but are completely devoid of coloured carotenoids. 'The infra-red spectrum of cells of the blue-green mutant is markedly different from that of the wild type and of the other photosynthetic mutants, despite the fact that its chlorophyll is chemically identical with theirs.

In his classical account of the Athiorhodaceae, van Niel (1944) devoted only one paragraph to the question of variations that affect photosynthetic ability. After describing the gradual weakening of photosynthetic power that occurs when aerobic representatives of the group are carried through many transfers in the dark, he concluded with the statement: 'So far, I have not observed a permanent loss of photosynthetic activity in cultures which have been grown in the dark for many years. Whether by careful selection subcultures might be isolated which have become non-photosynthetic remains a problem for the future.' Subsequent papers on the Athiorhodaceae contain no further information about this aspect of their biology. During the past ten years, however, many mutants with pigment systems differing from that of the wild type have been obtained in other photosynthetic organisms. Mutants of this kind in the green alga Chlorella have provided the material for studies by Granick and his collaborators on the biosynthesis of chlorophyll (summarized in Granick, 1954), and by Claes (1954) on carotenogenesis.

During work on the physiology of the non-sulphur purple bacterium Rhodopseudomonas spheroides, a spontaneous mutant differing from the wild type in its carotenoid pigments was discovered by Dr Germaine Cohen-Bazire. This observation prompted a more systematic study of mutational changes affecting the pigment system of $R$. spheroides. The results are reported in the present paper. 


\section{METHODS}

Biological material and media. The wild type employed was Rhodopseudomonas spheroides, strain 2.4.1, obtained from the collection of Professor C. B. van Niel of the Hopkins Marine Station of Stanford University. For the isolation of mutants, and for general purposes of cultivation under photosynthetic and non-photosynthetic conditions, the following medium (medium A) was used: ammonium malate $0.8 \mathrm{~g}$., magnesium sulphate 0.05 g., calcium chloride 0.005 g., yeast extract 1.0 g., and $100 \mathrm{ml} .0 .05 \mathrm{~m}$-potassium phosphate buffer $(\mathrm{pH} \mathrm{6 \cdot 8)}$. For solid media, $1 \cdot 0-1 \cdot 5 \%(\mathrm{w} / \mathrm{v})$ of agar was added. Cultures were maintained either photosynthetically as stabs incubated in the light, or aerobically as slopes incubated in the dark. For certain growth experiments, the partially defined medium described by Cohen-Bazire, Sistrom \& Stanier (1956) was used (medium B).

\section{Isolation of radiation-induced mutants}

Rhodopseudomonas spheroides was grown in medium $\mathbf{A}$ under semi-anaerobic conditions (completely filled Florence flasks) in the light. When the culture attained a population density of $2 \cdot 0-5 \cdot 0 \times 10^{9}$ organisms $/ \mathrm{ml}$. a sample was diluted 100 -fold, and $5 \mathrm{ml}$. of this diluted suspension were subjected to ultraviolet irradiation for $90 \mathrm{sec}$. from a General Electric Germicidal 8 W. lamp, held $14 \mathrm{in}$. above the Petri dish containing the suspension. Previous experiments showed that this treatment killed $99.9 \%$ of the organisms. The irradiated suspension was diluted to contain about 2000 viable organisms/ml., and $0.1 \mathrm{ml}$. of this dilution was spread over the surface of each of 50 plates of medium A. After 4-7 days of incubation under aerobic conditions in the dark, the plates were examined for pigment mutants.

\section{Spectrophotometric measurements}

A Beckman model DU spectrophotometer was used. The spectra of whole organisms were measured in a special cuvette holder with a pane of opal glass between the cuvettes and the photocell, which reduced the effects of scattering (Shibata, Benson \& Calvin, 1954). In order to facilitate comparison, the spectra of whole organisms were measured on suspensions adjusted to a fixed optical density at $680 \mathrm{~m} \mu$., since at this wavelength light absorption by the pigments is negligible.

\section{Isolation and determination of pigments}

Bacteriochlorophyll and bacteriophaeophytin. Bacteriochlorophyll was measured quantitatively as described by Cohen-Bazire et al. (1956). Bacteriophaeophytin was prepared by a modification of the method of van Niel \& Arnold (1938). The chlorophyll was extracted from $2-5 \mathrm{~g}$. (wet weight) of fresh organisms by two treatments with methanol, a total volume of $20 \mathrm{ml}$. being used. Ethyl ether $(20 \mathrm{ml}$.) was added, and the bacteriochlorophyll was then converted to bacteriophaeophytin by the addition of $2 \mathrm{ml} .5 \mathrm{~N}-\mathrm{H}_{2} \mathrm{SO}_{4}$. Twenty $\mathrm{ml}$. of water were added rapidly before the bacteriophaeophytin precipitated out of the acid methanol ether mixture. Bacteriophaeophytin and carotenoids 
went into the ether phase, which was washed to neutrality, dried, and evaporated to dryness. The residue was taken up in light petroleum $\left(60-70^{\circ}\right)$. To eliminate the carotenoids, the light petroleum extract was chromatographed on a column packed with a mixture of equal weights of $\mathrm{MgO}$ and Super $\mathrm{Cel}$; the column was then developed with an acetone+light petroleum mixture. Bacteriophaeophytin ran through the column rapidly and was collected in the effluent. In some cases, passage through a second column was required to eliminate a fastmoving yellow carotenoid.

Carotenoids. Coloured carotenoids were extracted from the finely ground, vacuum-dried organisms with carbon disulphide. This solvent extracts the carotenoids completely, but removes only a small amount of the chlorophyll. The extract was evaporated to dryness in vacuo and taken up in light petroleum. The pigments were separated on a magnesium oxide Super Cel $(1: 1)$ column, which was developed with $5 \%(\mathrm{v} / \mathrm{v})$ acetone in light petroleum. Where the separation was inadequate, purification was effected on a calcium hydroxide column, developed with $2 \cdot 5 \%(\mathrm{v} / \mathrm{v})$ acetone in light petroleum. The spectra were determined in light petroleum.

Phytoene. Fresh organisms were extracted twice with a mixture of 7 vol. acetone +1 vol. methanol. The pigments were transferred to a purified light petroleum by the addition of an equal volume of water. The commercial light petroleum used contained substances with a strong ultraviolet light absorption, which interfered with the spectrophotometry of phytoene. Special light petroleum, freed of these substances by passage through a silica gel column, was used in all work with phytoene. Acetone and methanol were removed by washing the light petroleum layer four times with a saturated aqueous solution of sodium chloride and once with water. The use of sodium chloride in the initial washes diminished the formation of emulsions. The extract was dried, concentrated to a small volume, and placed on a short $\mathrm{MgO}:$ Super Cel (1:1) column. The chromatogram was developed with light petroleum. Chlorophyll and its degradation products were held at the top of the column, while the phytoene ran through rapidly and was collected in the first 10-15 ml. of effluent. The spectrum was measured in light petroleum. Quantitative estimations of phytoene were based on the absorption at $286 \mathrm{~m} \mu$., using the value of 850 reported by Rabourn, Quackenbush \& Porter (1954) for the extinction coefficient $\left(\boldsymbol{E}_{1} \%\right.$ om. $)$.

\section{Preparation and fractionation of bacterial extracts}

Organisms were harvested, washed in 0.02 M-potassium phosphate buffer (pH 7.5), and resuspended in the same buffer. The suspension was subjected to sonic oscillation in a Raytheon 9-Kc Magnetostriction Oscillator for $10 \mathrm{~min}$. to break the organisms and release the chromatophores. Whole organisms and debris were removed by low-speed centrifugation $(10,000 \mathrm{~g})$ for $10 \mathrm{~min}$.; the resulting clarified extract was then further separated into a chromatophore fraction and a supernatant frection by centrifugation for $1 \mathrm{hr}$. at $144,000 \mathrm{~g}$. For the determination of the spectra of chromatophores, this fraction was resuspended in $0.05 \mathrm{~m}$-potassium phosphate buffer $(\mathrm{pH} 7 \cdot 5)$. 
Protein determinations. A sample of bacterial suspension which contained about $1.0 \mathrm{mg}$. protein was used. The organisms were sedimented, resuspended in $0.1 \mathrm{ml}$. water and extracted with $4.9 \mathrm{ml}$. of a $7: 2$ acetone : methanol mixture to remove the pigments which interfered with the colorimetric protein determination. After centrifugation, the extract was discarded and the protein content of the pellet determined by the biuret method (Weichselbaum, 1946).

\section{RESULTS}

Pigments of the wild type

Three principal pigments are associated with the photosynthetic apparatus of Rhodopseudomonas spheroides: bacteriochlorophyll, the form of chlorophyll found in all purple bacteria (van Niel, 1944); two carotenoids, one yellow and one red (van Niel, 1947). The colour of the organisms varies from brown to bright red, depending on the ratio between the two carotenoids, which changes

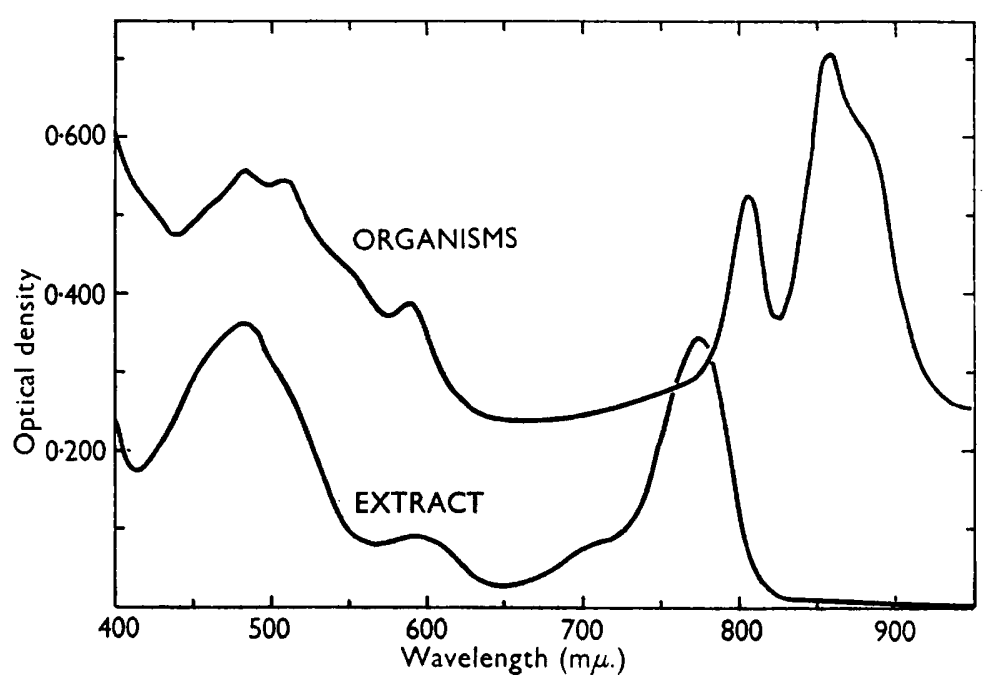

Fig. 1. A comparison of the absorption spectra in vivo and in vitro of the pigments in the wild type of Rhodopseudomonas spheroides. The curve marked organisus was measured on an aqueous suspension of whole organisms, with opal glass to minimize the effects of light scattering. The curve marked ExTract was measured on a methanol+acetone extract which contained all the bacteriochlorophyll and carotenoids, and was adjusted to the same pigment concentration as the cell suspension.

with the conditions of cultivation. Microscopic examination fails to reveal any intracellular localization of the photosynthetic pigments; but in extracts of the organisms, the pigments are found exclusively associated with a sedimentable fraction consisting of particles about $50 \mathrm{~m} \mu$. in. diameter. These particles, which have been termed ' grana' (Thomas, 1952) and ' chromatophores' (Pardee, Schachman \& Stanier, 1952), are presumably the functional equivalents of chloroplasts, although much smaller and apparently of simpler structure.

Fig. 1 shows the absorption spectrum of a suspension of living organisms, 
and of the mixed pigments extracted from these organisms by a mixture of methanol and acetone. In the organisms, light absorption by bacteriochlorophyll occurs principally in the infra-red region between 800 and $950 \mathrm{~m} \mu$., with peaks at 805 and $855 \mathrm{~m} \mu$. and a shoulder at about $875 \mathrm{~m} \mu$. There is a minor chlorophyll peak at $590 \mathrm{~m} \mu$, and a rising absorption at the short end of the visible spectrum which culminates in the Soret peak at $375 \mathrm{~m} \mu$. The region of carotenoid absorption in the organisms lies between 425 and $575 \mathrm{~m} \mu$., with peaks about 452, 477 and $508 \mathrm{~m} \mu$. It can be seen from Fig. 1 that the positions of the absorption maxima in the spectrum of the extracted pigments are markedly different from the positions of the maxima in the spectrum of the organisms. The spectrum of bacteriochlorophyll shows the greatest displacements: in organic solvents, it has virtually no absorption beyond $800 \mathrm{~m} \mu$., the main peak being situated at about $775 \mathrm{~m} \mu$., some $80 \mathrm{~m} \mu$. short of its position as seen in the organisms. Such major spectral shifts following extraction occur with the pigments of all purple bacteria. In describing the phenotype of Rhodopseudomonas spheroides with respect to the pigment system, both the determination of the spectrum of the whole organisms and the characterization of the isolated pigments have proved useful; certain of the changes which occur in mutants can be detected by one method, but not by the other.

The pigment system of Rhodopseudomonas spheroides is profoundly affected by the conditions under which the organisms are grown (Cohen-Bazire et al. 1956). Maximal concentrations of the photosynthetic pigments occur in organisms cultivated anaerobically in the light. Under such conditions, the pigment concentration is inversely related to light intensity. Furthermore, light intensity affects the shape of the infra-red spectrum: in organisms grown at low light intensities the absorption at $855 \mathrm{~m} \mu$. is far higher than at $875 \mathrm{~m} \mu$.; but as the light intensity during cultivation is increased, the shoulder at $875 \mathrm{~m} \mu$. becomes more and more prominent, eventually emerging as a separate peak at very high light intensities. Oxygen almost completely inhibits synthesis of the photosynthetic pigments, organisms grown in strict aerobiosis being practically colourless. Despite this, colonies on aerobic plates eventually show considerable pigmentation in the central regions, a phenomenon no doubt ascribable to the fact that in a well-developed colony many of the organisms are effectively shielded from contact with oxygen by their neighbours. In addition to suppressing pigment synthesis, oxygen has a special effect on the carotenoids of $R$. spheroides. The proportion of the red carotenoid is always much higher in organisms exposed to air than in organisms grown under strict anaerobiosis. Hence colonies or flask cultures exposed to air are red, whereas stabs or bottle cultures are brown. When an anaerobically grown culture is exposed to air, it turns red in a short period of time. A quantitative study of the pigment changes that occur (van Niel, 1947) strongly suggests that there is a stoichiometric conversion of the yellow carotenoid to the red one, although this conclusion has been questioned recently (Goodwin, Land \& Osman, 1955).

It is clear from this discussion that an accurate characterization of the phenotype with respect to pigmentation is possible only when the organisms are grown under closely controlled and reproducible conditions. Unless otherwise 
specified, all reported data on pigments have been obtained with organisms grown anaerobically (atmosphere: $95 \% \mathrm{~N}_{2}+5 \% \mathrm{CO}_{2}$ ) in medium $\mathrm{A}$ at a temperature of $30^{\circ}$ and at a uniform light intensity close to that saturating for growth, and harvested during the exponential phase of growth. These conditions cannot be used, of course, for the cultivation of non-photosynthetic mutants, which were grown at $30^{\circ}$ semi-aerobically in the dark in medium $A$.

\section{Spontaneous variation in the wild type}

Cultures of the wild type maintained anaerobically in the light (i.e. under conditions where photosynthesis is obligatory) show little tendency to produce spontaneous pigment mutants. On two occasions, however, spontaneous mutants of the green phenotype, which is described in a later section, were isolated from photosynthetic cultures of the wild type. Since these mutants have the same photosynthetic growth rate as the wild type, they are presumably not eliminated by selection in anaerobiosis and light.

Grown aerobically in the dark, the wild type is far less stable. After repeated transfers under these conditions, two mutants make their appearance. One produces colonies which are much paler than those of the wild type, while the other produces colourless colonies. The former can still grow photosynthetically: the latter cannot, but will revert to the wild type again when placed under photosynthetic growth conditions. A similar pattern of genetic instability during aerobic growth in the dark is shared by the various classes of photosynthetic mutants to be described below. We have not made a detailed study of these spontaneous variants. Presumably during aerobic growth, mutants with a lessened ability to manufacture the photosynthetic pigments are not eliminated (and perhaps even favoured) by selection, thus gradually accumulating in the population. In so far as they have been examined, the pale phenotypes do not differ qualitatively from their parental stocks with respect to their pigments. This type of variation no doubt explains the physiological observations made by van Niel (1944):

'Aerobic representatives may, of course, be kept on ordinary slants and grown in the dark. However, it is my impression that under such conditions the photosynthetic ability of the organisms slowly weakens. I have had cultures of a number of strains both in stabs, exposed to continuous illumination, and on slants which were regularly kept in the dark except at times when they were transferred. In the course of ten to fifteen years the 'dark cultures', though still capable of slow and scanty development in the light, were decidedly less suitable for photosynthesis experiments than the corresponding stab cultures. By a process of selection one can succeed in gradually restoring the original vigour, but this takes time, and many transfers in media where growth depends on photosynthetic activity are required to achieve it.'

The isolation of induced mutants

On aerobic plates spread with irradiated suspensions and incubated in the dark at $30^{\circ}$, the central portions of the colonies have become sufficiently pigmented after 4-7 days to permit visual discrimination between colonies of 
the mutants and of the wild type. In all, about 50 mutants were isolated. On the basis of their colour, they were divided into five main phenotypes: dark red, brown, green, blue-green and colourless. Mutants of the green phenotype were most common, followed in order by dark red, brown, blue-green and colourless. All save those of the colourless phenotype proved to be still capable of photosynthetic growth, and a more refined specification of the phenotypes could then be made by comparing the absorption spectra of the organisms, grown anaerobically in the light, with the spectrum of the wild type organisms grown under the same standard conditions; Fig. 2 shows some representative spectra. That of the dark red phenotype is not included, since the spectrum of these mutants does not differ detectably from that of the wild type.

In organisms of the brown and green phenotypes, the chlorophyll peaks $(375,590,805,855 \mathrm{~m} \mu$.) are identical with those of the wild type. In the region of carotenoid absorption, the brown phenotype shows peaks at 430,459 and $491 \mathrm{~m} \mu$., all displaced to considerably shorter wavelengths than those of the wild type. The peaks in the carotenoid region of the spectrum of the green phenotype lie close to those of the brown, at 430,457 and $488 \mathrm{~m} \mu$.; but the relative heights are very different, and there is also a marked decrease in absorption around $550 \mathrm{~m} \mu$. The spectrum of the blue-green phenotype differs from that of the wild type in the regions of both chlorophyll and carotenoid absorption. Chlorophyll peaks at 375 and $590 \mathrm{~m} \mu$. are still present, but in the infra-red region the peak of the wild type at $805 \mathrm{~m} \mu$. has been replaced by a long shoulder around $810 \mathrm{~m} \mu$., and the major peak of the wild type at $855 \mathrm{~m} \mu$. has disappeared, giving place to a completely symmetrical peak with a maximum at $877 \mathrm{~m} \mu$. There are no peaks whatsoever in the carotenoid region, and the general lowering of the absorption suggests the total absence of carotenoid pigments. In shape (though not in the absolute positions of the peaks), the spectrum of the blue-green phenotype bears a considerable resemblance to that of pure bacteriochlorophyll in organic solvents.

Lastly, the spectrum of a representative of the colourless phenotype (grown semi-aerobically in the dark) is shown in Fig. 2. There are no characteristic peaks in the regions either of chlorophyll or of carotenoid absorption; the small isolated peak at $420 \mathrm{~m} \mu$. probably reflects cytochrome absorption.

In order to determine the intracellular localization of the pigments in the various photosynthetic mutants, cell extracts were made, the chromatophore fractions were isolated by differential centrifugation, and spectra were measured on these fractions. The chromatophore suspensions had spectra virtually identical, both in general shape and in the position of the maxima, with those of the organisms from which they were prepared. It thus appears that the changes in the pigment systems of the mutants have occurred within the chromatophores.

\section{Identification of pigments in the photosynthetic mutant phenotypes}

A single strain of each photosynthetic phenotype was selected for chemical study of the pigments and comparison with those of the wild type.

Chlorophyll. Since bacteriochlorophyll is somewhat unstable, it was identi- 
fied by the preparation of the phaeophytin derivative, as described in Methods. The spectra in chloroform of the phaeophytins prepared from the wild type, and from the dark red, brown, green and blue-green mutants were identical. Furthermore, they coincided with the spectrum of purified bacteriophaeophytin

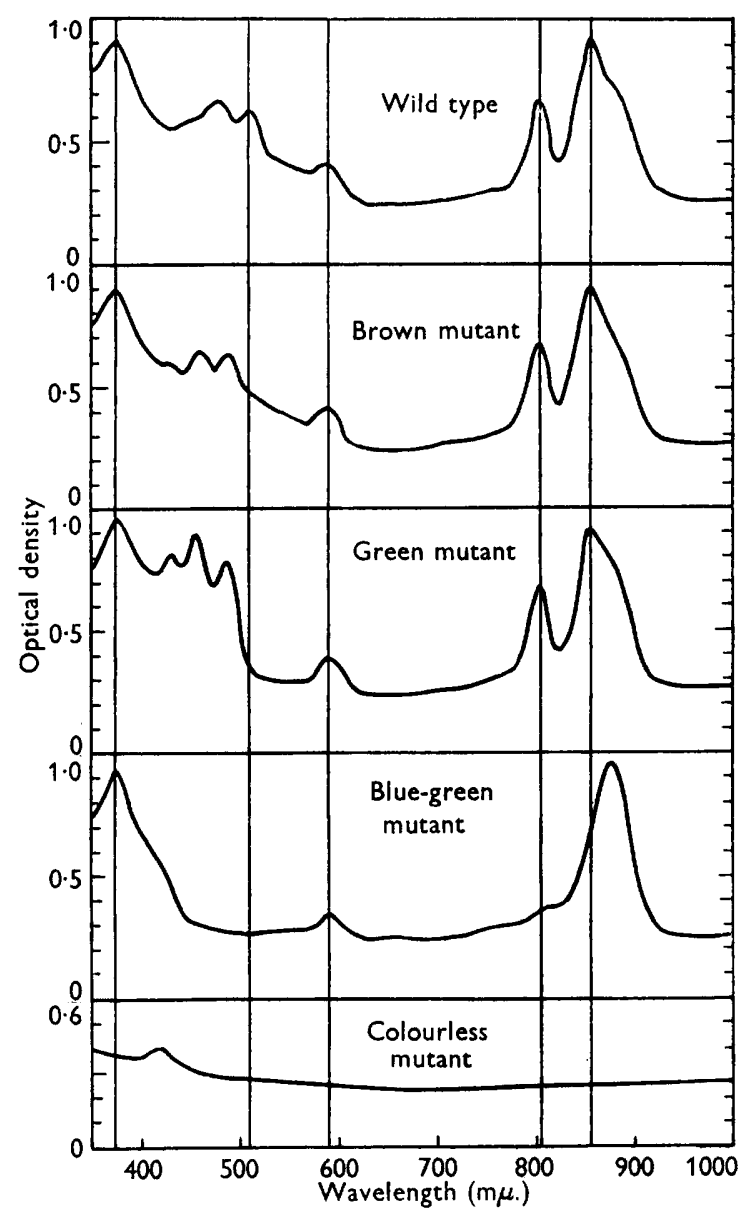

Fig. 2. Absorption spectra of whole organisms of the various phenotypes of Rhodopseudomonas spheroides. All spectra were adjusted to the same optical density at $680 \mathrm{~m} \mu$. Vertical lines have been drawn through some of the maxima in the spectrum of the wild type to facilitate comparison.

from Rhodospirillum rubrum. Since the only change that occurs in the conversion of a chlorophyll to a phaeophytin is the elimination of magnesium from the molecule, it seems evident that all the strains examined contained bacteriochlorophyll. This might have been anticipated for the dark red, brown and green mutants, whose cells show the same chlorophyll absorption as does the wild type. However, as mentioned above, the infra-red spectrum of the blue-green mutants differs markedly from that of the wild type. The identity of the chlorophyll in this mutant was therefore tested more directly. The 
organisms were extracted with methanol, and the spectrum of the methanolic extract was compared with that of pure bacteriochlorophyll in the same solvent. Since the blue-green rnutant contains no coloured carotenoids, a chromatographic purification of the methanolic extract was unnecessary for spectral comparison. The extract showed complete spectral identity with bacteriochlorophyll over the range from 350 to $800 \mathrm{~m} \mu$. It may therefore be concluded that the anomalous infra-red spectrum of the blue-green mutant is not caused by a change in the chemical nature of its chlorophyll.

Coloured carotenoids. The carotenoids in mutants of the green, brown and dark red phenotypes were isolated as described in Methods and compared with those of the wild type. All the spectra (Fig. 3) were measured in light petroleum,

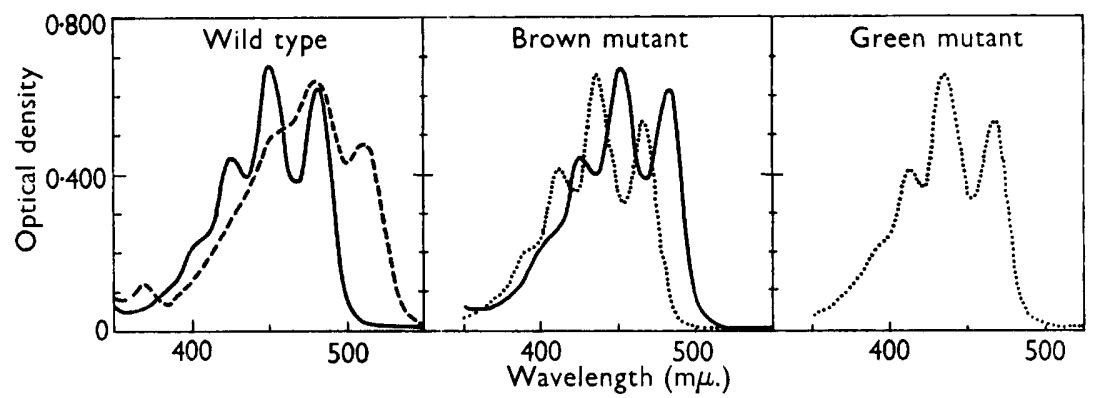

Fig. 3. Absorption spectra, measured in light petroleum, of the principal carotenoid pigments present in the wild-type and in the green and brown mutants of Rhodopseudomonas spheroides. Solid line=yellow pigment of wild type. Dashed line: red pigment of wild type. Dotted line=yellow pigments of mutant (neurosporene and dihydroxyneurosporene).

and the reported absorption maxima refer to this solvent. The yellow carotenoid of the wild type had three very sharp peaks at 426,451 and $481 \mathrm{~m} \mu$; the red carotenoid of the wild type had relatively broad peaks at 481 and $512 \mathrm{~m} \mu$., together with a shoulder at $451 \mathrm{~m} \mu$.

The green mutant contained neither pigment of the wild type, but instead two new yellow carotenoids, present in approximately equal amounts. These pigments have identical spectra, similar in shape to that of the yellow pigment from the wild type, but with peaks at 413,437 and $467 \mathrm{~m} \mu$. They are separable from one another owing to their markedly different rates of movement on columns. Nakayama \& Chichester (unpublished studies) have crystallized both these substances, and find that their specific extinction coefficients are slightly different. The one that moves most rapidly appears to be identical with neurosporene, a carotenoid found in Neurospora sp. by Haxo (1949); the other is a dihydroxy derivative of neurosporene. Nakayama \& Chichester have also found very small traces of neurosporene in extracts of the wild type organisms.

The brown mutant contains the yellow carotenoid of the wild type and the more rapidly moving of the two carotenoids found in the green mutant (neurosporene). In addition, very small quantities of the red carotenoid of the wild type are present. 
The dark red mutant contains both the carotenoids of the wild type, together with traces of a second red carotenoid, which moves very slowly on columns, even when a $1: 1$ mixture of acetone + light petroleum is used for development. This pigment has a spectrum similar in shape to that of the wild-type red carotenoid, but with maxima at 462, 490 and $522 \mathrm{~m} \mu$. It has not been obtained in sufficient quantities for further characterization.

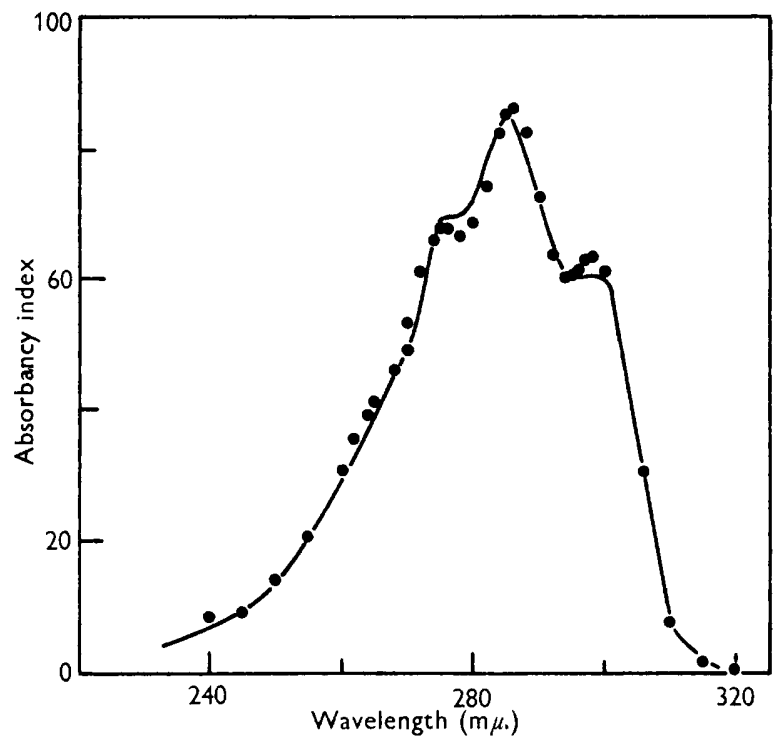

Fig. 4. Absorption spectrum of crude phytoene extracted from the blue-green mutant of Rhodopseudomonas spheroides, compared with that of purified phytoene from tomato fruit. Both curves adjusted to the same optical density at $285 \mathrm{~m} \mu$. Solid line $=$ purified phytoene from tomato (data of Dr J. W. Porter). Points = phytoene of the blue-green mutant of $\boldsymbol{R}$. spheroides. According to Dr Porter (personal communication), the secondary maxima at 275 and $298 \mathrm{~m} \mu$. in crude phytoene from tomato are considerably more pronounced than in the purified material. Hence the differences in this respect shown in the graph probably reflect the fact that the bacterial phytoene has not been purified.

Colourless polyenes. Chromatographic analysis of an extract of the pigments from the blue-green mutant confirmed the absence of coloured carotenoids, already strongly suggested by the absorption spectrum of the organisms. Apart from bacteriochlorophyll and its degradation products, the only coloured band on the column was a faint yellow one with a single symmetrical peak at $280 \mathrm{~m} \mu$. and a slowly decreasing absorption in the visible region between 400 and $500 \mathrm{~m} \mu$. This substance has not been identified, but from its spectrum it is evidently not a carotenoid. When effluents from columns treated with extracts of the blue-green mutant were examined spectrophotometrically, large amounts of a colourless non-fluorescent substance with a strong absorption in the ultraviolet were found; all of this material was contained in the first $10-15 \mathrm{ml}$. of light petroleum which emerged from the column. The behaviour of this material on the column and its absorption spectrum (Fig. 4) suggest that it is phytoene, 
a $\mathrm{C}_{40}$ polyene originally fourd by Porter \& Zscheile (1946) in tomato fruits. The related colourless polyene, phytofluene (Wallace \& Porter, 1952) does not appear to occur in the blue-green mutant; it is a fluorescent substance, and the chromatograms of extracts from the blue-green mutant do not show any colourless fluorescent bands.

Since an absorbancy index for phytoene has been published (Rabourn et al. 1954), it was possible to determine roughly the amount of phytoene present in the blue-green mutant, by measuring the total ultraviolet absorption of the effluents from columns treated with extracts prepared from a known quantity of cell material. Such determinations show the presence of 1-3 mg. phytoene/g. bacterial protein in photosynthetically-grown organisms. This is of the same order of magnitude on a weight basis as the total quantity of coloured carotenoids in organisms of the wild type grown under similar conditions. Examination of the appropriate fraction from columns treated with extracts of the pigments from wild type organisms failed to reveal any phytoene (limits of detection; about $1 \%$ of the concentration present in the blue-green mutant). Goodwin et al. (1955) reported the absence of phytoene from organisms of the wild type.

\section{Localization of phytoene in the blue-green mutant}

Phytoene cannot be directly detected by the spectrophotometric examination of whole organisms or chromatophores, since its absorption spectrum is masked by scattering and by the ultraviolet absorption of the nucleic acids and proteins. A special experiment was accordingly carried out in order to ascertain its intracellular distribution in the blue-green mutant. A sample of washed organisms was ground with alumina, and after low-speed centrifugation of the resulting extract to remove alumina and coarse debris, the chromatophores and the soluble fraction were isolated by differential high-speed centrifugation. The amounts of protein, chlorophyll and phytoene in these fractions and in an equivalent sample of the original suspension of organisms were then determined. The recoveries of the fractions from the cell extract were far from quantitative, and hence for purposes of comparison the percentage recovery of each fraction had to be calculated by indirect means. No chlorophyll occurred in the soluble fraction; therefore the recovery of the chromatophore fraction could be estimated by comparing the total chlorophyll content of the isolated chromatophores with the chlorophyll content of the original suspension of organisms. This calculation showed a chromatophore recovery of $16.4 \%$. By applying the same correction factor to the protein content of the isolated chromatophores $(49 \mathrm{mg}$.), it was calculated that the original total protein content of the chromatophore fraction was $297 \mathrm{mg}$. Subtracted from the total protein content of the organisms (786 mg.), this gives a value of $489 \mathrm{mg}$. for the protein of the organisms not in the chromatophores. The total protein content of the isolated soluble fraction was $225 \mathrm{mg}$., and on the assumption that 'protein not in chromatophores' was all in this fraction, the recovery of the soluble fraction was $45 \%$. By applying these correction factors for recovery of two cell-fractions to the determined phytoene values, one can obtain an approxi- 
mate indication of the intracellular distribution of phytoene (Table 1 ). The calculated recovery of phytoene was $77 \%$ of that present in the whole organisms, and of this, over $90 \%$ was in the chromatophore fraction. It must be admitted that the experiment is a highly inaccurate one; however, a second type of calculation substantiated the general conclusion that the phytoene is concentrated in the chromatophores. This is the calculation of the phytoene: protein ratios, shown in Table 2 ; in whole organisms, this ratio is $\mathbf{2 \cdot 8 2}$. It increases in the chromatophore fraction to $\mathbf{5 \cdot 1 0}$, and decreases in the soluble fraction to $0 \cdot 31$.

Table 1. Intracellular distribution of phytoene in the blue-green mutant of Rhodopseudomonas spheroides

\begin{tabular}{|c|c|c|c|c|}
\hline Material & $\begin{array}{c}\text { Recovery } \\
\text { of fraction } \\
(\%)\end{array}$ & $\begin{array}{l}\text { Phytoene } \\
\text { content } \\
\text { (mg.) }\end{array}$ & $\begin{array}{l}\text { Phytoene } \\
\text { content, } \\
\text { corrected for } \\
\text { recovery } \\
\text { (mg.) }\end{array}$ & $\begin{array}{c}\text { Phytoene, } \\
\text { amount in } \\
\text { whole } \\
\text { organisms } \\
(\%)\end{array}$ \\
\hline Whole organisms & 100 & $2 \cdot 22$ & $2 \cdot 22$ & 100 \\
\hline Chromatophores & 16 & $0 \cdot 25$ & 1.54 & 70 \\
\hline Soluble fraction & 45 & 0.07 & $0 \cdot 16$ & 7 \\
\hline
\end{tabular}

Although both calculations show that most of the phytoene is in the chromatophores, a detectable amount was none the less found in the soluble fraction. This may have resulted from a partial disintegration of chromatophores during the preparation or handling of the cell-free extract; but the absence of chlorophyll from the soluble fraction makes this interpretation somewhat unlikely. Accordingly, it is possible that a small part of the phytoene occurs in the organisms unattached to chromatophores.

Table 2. Phytoene-protein ratios in organisms, chromatophores and soluble fraction of the blue-green mutant of Rhodopseudomonas spheroides

\begin{tabular}{lccc}
\multicolumn{1}{c}{$\begin{array}{c}\text { Phytoene } \\
\text { content } \\
\text { Material }\end{array}$} & $\begin{array}{c}\text { Protein } \\
\text { content } \\
(\mathrm{mg})\end{array}$ & $\begin{array}{c}\text { Phytoene, } \\
\text { mg./g. protein }\end{array}$ \\
Whole organisms & $\mathbf{2 \cdot 2 2}$ & $\mathbf{7 8 6}$ & $\mathbf{2 \cdot 8 2}$ \\
Chromatophores & $\mathbf{0 . 2 5}$ & 49 & $5 \cdot 10$ \\
Soluble fraction & $\mathbf{0 . 0 7}$ & $\mathbf{2 2 5}$ & $\mathbf{0 \cdot 3 1}$
\end{tabular}

\section{Biological observations on the mutants}

Green phenotype. Four induced and two spontaneous mutants of this class were isolated. Numerous kinetic experiments have been conducted with one of the spontaneous green mutants (Cohen-Bazire et al. 1956), whose growth rate proved to be identical with that of the wild type under all environmental conditions; less detailed observations on the other strains indicate that the same thing may be true for the class as a whole. Accordingly, the change in carotenoid constitution does not seem to affect the gross photosynthetic ability. Most strains of this phenotype are genetically stable when maintained 
anaerobically in the light; two strains have shown occasional reversions to the wild type.

Brown phenotype. Three induced mutants of this class were isolated. They grow well under photosynthetic conditions, but systematic comparisons with the growth rate of the wild type have not so far been made. Cultures maintained anaerobically in the light give rise to occasional paler brown mutants, but reversion to the wild type has never been observed.

Dark red phenotype. Numerous induced mutants of this class were isolated, but genetic instability made their maintenance exceedingly difficult. They grow well under photosynthetic conditions, but readily give rise to new mutant types with little or no photosynthetic ability, as well as reverting to wild type.

Blue-green phenotype. Four induced mutants of this class were isolated. They can grow photosynthetically, but the rate is never as great as that of the wild type. Originally all members of the group were very unstable when grown anaerobically in the light, mass reversion to the wild type occurring after one or two transfers. As a result of repeated selection over the past 12 months, one strain has now been stabilized, and can often be carried through several transfers under photosynthetic conditions without reversion. When grown aerobically in the dark, the blue-green mutants do not revert, but tend, sooner or later, to lose their photosynthetic ability as a result of the appearance of weakly pigmented or unpigmented secondary mutants. The safest method of maintenance for mutants of this class is, therefore, cultivation on slopes in the dark, alternated with a periodic transfer through a flask culture incubated in the light (i.e. under photosynthetic growth conditions), followed by streaking and selection of a typical colony, from which a new series of transfers on slopes is initiated.

Photosynthetically-grown cultures of this phenotype have a bright bluegreen colour. On aerobic plates, however, the characteristic pigmentation is less easily seen; the colonies are a very pale greyish green, difficult to distinguish without practice from those of colourless mutants. A detailed study of the biology of one of the blue-green mutants is reported elsewhere (Sistrom, Griffiths \& Stanier, 1956).

Colourless phenotype. Six induced mutants of this class were isolated. The class is a provisional one, and its designation is somewhat of a misnomer, since the colour of the organisms actually varies from white to a deep tan or brownish green in the different strains included. What is characteristic of all members is the absence of chlorophyll and carotenoid peaks in the spectra of the organisms, coupled with inability to grow photosynthetically. When placed under photosynthetic growth conditions, three strains revert to the wild type. In the absence of reversion, the identity of the remaining three strains as mutants of Rhodopseudomonas spheroides is not absolutely certain; but in morphology and colony form they closely resemble the wild type. Biochemical studies, so far not undertaken, may well show that this class includes several different phenotypes. 


\section{DISCUSSION}

\section{The colourless mutants}

Most of the mutant phenotypes isolated from Rhodopseudomonas spheroides differ from the wild type with respect to pigmentation only in their carotenoid content. An exception is the group of colourless mutants, which have lost both bacteriochlorophyll and carotenoids. Since some of these mutants revert readily to the wild type, the simultaneous loss of chlorophyll and carotenoids from the organism can presumably take place in a single mutational step. Several possible mechanisms can be envisaged. First, it is conceivable that the ability to elaborate chromatophores or the structure of the chromatophore itself may be affected. Secondly, both classes of photosynthetic pigments may share a common biosynthetic origin, the mutational block in the colourless mutants occurring before the branch point. This interpretation need not necessarily imply a common pathway for porphyrin and carotenoid synthesis, since a common block in the synthesis of carotenoids and of the phytol sidechain of chlorophyll would no doubt be effective in preventing chlorophyll synthesis. The third possibility is that the colourless mutants are blocked genetically in the pathway of chlorophyll synthesis, this entailing as a secondary and indirect consequence the failure of carotenogenesis. It should be recalled that owing to their inability to photosynthesize, these mutants can be grown only in the presence of oxygen, which effectively decreases the synthesis of both chlorophyll and carotenoids in the wild type. Whatever the explanation, these mutants prove that non-photosynthetic variants of the Athiorhodaceae can exist, thus answering the question posed in 1944 by van Niel.

\section{The chain of carotenoid synthesis in the photosynthetic mutants}

Table 3 summarizes the distribution of carotenoids in the wild type and in the brown, green and blue-green mutants. With the exception of the dark red mutant, which contains both wild-type carotenoids together with small quantities of a new red pigment, all the photosynthetic mutants are characterized by the accumulation of carotenoids which absorb light at shorter wavelengths than do those of the wild type; the extreme instance of this behaviour is shown

Table 3. The distribution of carotenoids in Rhodopseudomonas spheroides and some of its mutants

$\begin{array}{lccccc}\text { Phytoene } & \begin{array}{c}\text { Mutant } \\ \text { yellow } \\ (\text { slow } \dagger\end{array} & \begin{array}{c}\text { Mutant } \\ \text { yellow } \\ \text { (fast) }\end{array} & \begin{array}{c}\text { Wild-type } \\ \text { Yellow }\end{array} & \begin{array}{c}\text { Wild-type } \\ \text { Red }\end{array} \\ \text { Wild type } & (297 \mathrm{~m} \mu .)^{*} & (466 \mathrm{~m} \mu .) & (466 \mathrm{mu} .) & (481 \mathrm{~m} \mu .) & (512 \mathrm{~m} \mu .) \\ \text { Brown } & - & - & \text { Trace } & + & + \\ \text { Green } & - & - & + & + & \text { Trace } \\ \text { Blue-green } & - & + & + & - & - \\ & + & - & - & - & -\end{array}$

* Figures in parentheses are the values for the longest absorption maximum of the pigment, in light petroleum.

$\dagger$ Dihydroxyneurosporene.

$\$$ Neurosporene. 
by the blue-green mutant, which contains no coloured carotenoids, and accumulates instead phytoene, a colourless polyene whose light absorption occurs exclusively in the ultraviolet region. The shift in spectrum shows that the carotenoids of the mutants contain less extensive systems of conjugated double bonds than do those of the wild type. If we interpret these mutants in the customary fashion, as strains in which the normal pathway of biosynthesis has been blocked at specific points by derangements of the relevant enzyme systems, the results are in general agreement with the mechanism of carotenogenesis proposed by Porter \& Lincoln (1950). As a result of their studies on the distribution of carotenoids and colourless polyenes in tomato fruits of different genotypes, these authors postulated that the penultimate stages of carotenoid formation involve stepwise dehydrogenations of originally highly saturated open-chain $\mathrm{C}_{\mathbf{4 0}}$ hydrocarbons:

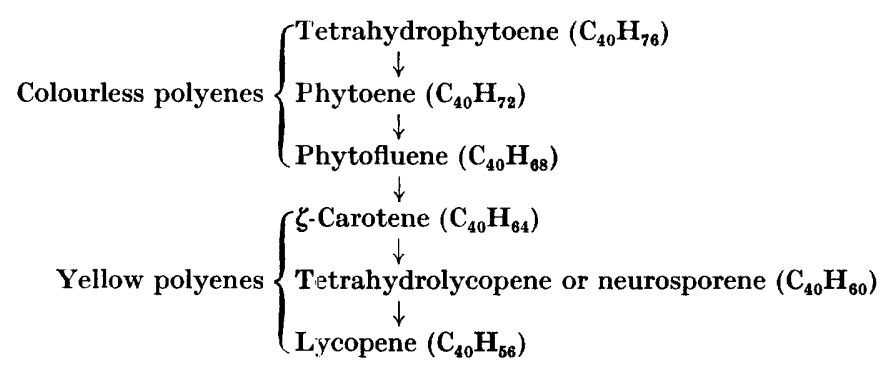

Other carotenoid pigments could be derived from the later members of this series by further dehydrogenation, ring closure and the introduction of oxygen atoms. The Porter-Lincoln series has been greeted with considerable scepticism by other workers on carotenoids (e.g. Mackinney, 1952; Goodwin, 1952, 1955); it remains, however, the only general hypothesis of carotenogenesis so far supported by any evidence. One of the greatest weaknesses of the series as originally postulated was the fact that the structures of its more saturated members were far from established; indeed, later work suggests that phytoene is an octahydrolycopene, and that phytofluene may also be at this oxidation level, differing from phytoene in its conjugation system (see Goodwin, 1955, for a discussion). Thus at present the sequential relationships in the series are far from clear. None the less, the general concept implicit in the PorterLincoln series may be correct. Its predictions have been borne out by recent work on mutants of Chlorella (Claes, 1954), which showed that blocks in normal carotenogenesis caused accumulations of the more saturated members of the Porter-Lincoln series; and as mentioned above, our findings with Rhodopseudomonas spheroides also fit the general concept.

The pattern of distribution of carotenoids in the mutants of Rhodopseudomonas spheroides can be explained in terms of the following tentative pathway:

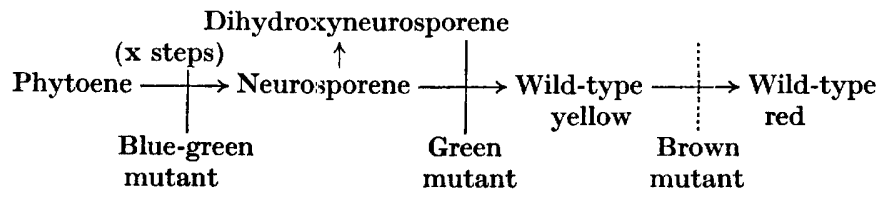


The brown mutant accumulates approximately equal amounts of neurosporene and of the wild-type yellow pigment, together with traces of wild-type red. These facts suggest that it is almost completely blocked in the performance of the normal yellow $\rightarrow$ red conversion, and that the resulting build-up of the normal yellow pigment causes a substantial accumulation of its immediate precursor, neurosporene. This interpretation is supported by the fact that traces of neurosporene occur in the wild type. The very close spectral similarity of the normal yellow pigment to neurosporene indicates that it has a similar structure, and fits the notion of a close biosynthetic relationship.

The green mutant accumulates a mixture of neurosporene and dihydroxyneurosporene, but neither of the wild-type pigments. This fact suggests that it is completely blocked in the conversion of neurosporene to the wild-type yellow pigment. Dihydroxyneurosporene might be either a precursor of neurosporene or a side-product; we have shown it in the latter role.

Lastly, the blue-green mutant, which accumulates exclusively phytoene in quantities roughly equivalent to the quantities of pigments present in wildtype cells, is most easily interpreted as having a complete block in the main pathway of carotenogenesis immediately after phytoene. There are possibly several steps between phytoene and neurosporene; if so, further work should reveal the existence of additional mutant classes with blocks in this region of biosynthesis.

One of the arguments that has been used against the Porter-Lincoln scheme is the failure to detect colourless polyenes such as phytoene or phytofluene in many organisms and tissues which contain carotenoid pigments. However, biosynthetic intermediates may not necessarily be detectable in cell materials which contain the final products; the presence or absence of such intermediates will be governed by the relative rates of the successive biosynthetic stepreactions. The apparent absence of phytoene from cells of the wild type of Rhodopseudomonas spheroides, which was reported by Goodwin et al. (1955) and has been confirmed by us, is a case in point.

The blue-green mutant constitutes the first known exception to the rule that functional photosynthetic systems always contain carotenoid pigments in addition to chlorophyll (Strain, 1949). Despite the fact that coloured carotenoids have been entirely replaced by phytoene, this mutant is still capable of photosynthetic growth. However, detailed physiological studies (Sistrom et al., 1956) show that it has suffered certain functional derangements.

\section{The absorption spectrum of bacteriochlorophyll in Rhodopseudomonas spheroides}

The absorption spectra of bacteriochlorophyll in the green and brown mutants show no detectable changes from that in the organisms of the wild type; but an entirely new pattern of infra-red absorption is found in the blue-green mutant, despite the fact that its chlorophyll is chemically identical with the bacteriochlorophyll of the wild type.

It has long been known that the infra-red spectra of Rhodopseudomonas 
spheroides and other species of purple bacteria contain certain bands that have no counterparts in the spectrum of isolated bacteriochlorophyll. The comparison of the spectra of bacteriochlorophyll in vivo and in vitro is complicated by the shifts in the positions of the maxima which occur upon extraction, but on any scheme of assignment, certain of the bands observed in vivo remain unaccounted for in the spectrum in vitro. Consequently it has been generally assumed that within the organisms bacteriochlorophyll is conjugated with two or more proteins, these several chlorophyll-protein conjugates having different absorption maxima in the infrared region. On this interpretation of the normal infrared spectrum, the disappearance of the peaks at 805 and $855 \mathrm{~m} \mu$. in the infrared spectrum of the blue-green mutant would reflect the loss from the chromatophore of two proteins, which are conjugated with chlorophyll in wildtype organisms and the other photosynthetic mutants. Such an explanation cannot be ruled out, although it would necessitate a complex interpretation of the primary biochemical lesion in the blue-green mutant, which also evidently affects carotenogenesis. A mole attractive hypothesis is one which assumes that the change of infra-red absorption is a consequence of the elimination of coloured carotenoids from the chromatophore; several possible ways in which such an elimination might affect the infrared spectrum can be envisaged, and are discussed at greater length elsewhere (Calvin, 1955; Sistrom et al. 1956).

It is a pleasure to thank the following for their aid: Dr C. O. Chichester and $\mathrm{Mr}$ T. Nakayama for frequent elucidations of the theory and practice of carotenoidology; Mr E. J. Behrman for help in the original fractionation of the carotenoids from the mutants; and Dr J. W. Porter and J. H. C. Smith for furnishing unpublished reference spectra.

This work was supported by grants-in-aid from the National Science Foundation and from the American Cancer Society, upon recommendation of the Committee on Growth of the National Research Council.

\section{REFERENCES}

Calvin, M. (1955). A contribution to the problem of carotenoid function in photosynthesis. Nature, Lond. 176, 1215.

Clates, H. (1954). Analyse der biochemischen Synthesekette für Carotinoide mit Hilfe von Chlorella-Mutanten. Z. Naturf. 9 B, 461.

Cohen-Bazire, G., Sistrom, W. R. \& Stanier, R. Y. (1956). Kinetic studies of pigment synthesis by non-sulfur purple bacteria. J.cell. comp. Physiol. (in the Press).

Goodwin, T. W. (1952). The Comparative Biochemistry of the Carotenoids. London: Chapman and Hall.

Goodwin, T. W. (1955). Carotenoids. Annu. Rev. Biochem. 24, 496.

Goodwin, T. W., Land, D. G. \& Osman, H. G. (1955). Studies in carotenogenesis. 14. Carotenoid synthesis in the photosynthetic bacterium Rhodopseudomonas spheroides. Biochem. J. 59, 491.

Granick, S. (1954). Metabolism of heme and chlorophyll. In Chemical Pathreays of Metabolism, 2, 287. New York: Academic Press.

Haxo, F. (1949). Studies on the carotenoid pigments of Neurospora. Arch. Biochem. Biophys. 20, 400.

Mackinney, G. (1952). Carotenoids. Annu. Rev. Biochem. 21, 473.

VAN NiEL, C. B. (1944). The culture, general physiology, morphology and classification of the non-sulfur purple and brown bacteria. Bact. Rev. 8, 1 . 
van Niel, C. B. (1947). Studies on the pigments of the purple bacteria. III. The yellow and red pigments of Rhodopseudomonas spheroides. Leeurvenhoek ned. Tijdschr. 12, 156.

van Niel, C. B. \& Arnold, W. A. (1938). The quantitative estimation of bacteriochlorophyll. Enzymologia, 5, 244.

Pardee, A. B., Schachman, H. K. \& Stanier, R. Y. (1952). Chromatophores of Rhodospirillum rubrum. Nature, Lond. 169, 282.

Ponter, J. W. \& Lincoln, R. E. (1950). The mechanism of carotene biosynthesis. Arch. Biochem. Biophys. 27, 398.

Porter, J. W. \& Zscheile, F. P. (1946). Naturally occurring colorless polyenes. Arch. Biochem. Biophys. 10, 547.

Rabourn, W. J., Quackenbush, F. W. \& Porter, J. W. (1954). Isolation and properties of phytoene. Arch. Biochem. Biophys. 48, 267.

Shibata, K., Benson, A. A. \& Calvin, M. (1954). The absorption spectra of suspensions of living micro-organisms. Biochim. biophys. Acta, 15, 461.

Sistrom, W. R., Griffiths, M. \& Stanier, R. Y. (1956). The biology of a photosynthetic bacterium which lacks coloured carotenoids. J. cell. comp. Physiol. (in the Press).

Strain, H. H. (1949). Function and Properties of the Chloroplast Pigments. In Photosynthesis in Plants, p. 133. Ames: Iowa State College Press.

Thomas, J. B. (1952). A note on the occurrence of grana in algae and photosynthesizing bacteria. Proc. Acad. Amst. C, 55, 207.

Wallace, V. \& Porter, J. W. (1952). Phytofluene. Arch. Biochem. Biophys. 36, 468.

Weichselbaum, T. E. (1946). An accurate and rapid method for the determination of proteins in small amounts of blood serum and plasma. Amer. J. clin. Path., tech. Suppl. 10, 40. 